\title{
Biocatalytic Reduction of Carbonyl Compounds by Actinobacteria from Two Genera of the Micromonosporaceae Family: Actinoplanes and Dactylosporangium
}

\author{
K. Ishihara ${ }^{\text {* }}$, K. Morita ${ }^{1}$, Y. Nishimori ${ }^{1}$, S. Okamoto ${ }^{1}$, T. Hiramatsu ${ }^{1}$, A. Ohkawa ${ }^{1}$, D. \\ Uesugi $^{2}$, M. Yanagi ${ }^{1}$, H. Hamada ${ }^{1}$, N. Masuoka ${ }^{3}$ and N. Nakajima ${ }^{4}$ \\ ${ }^{1}$ Department of Life Science, Okayama University of Science, Okayama, Japan \\ ${ }^{2}$ Department of Research \& Development, JO Cosmetics Co., Ltd., Tokyo, Japan \\ ${ }^{3}$ Department of Research \& Development, Institute for Fruit Juice Research in Tsudaka, Co., \\ Ltd., Okayama, Japan \\ ${ }^{4}$ Department of Nutritional Science, Okayama Prefectural University, Soja, Okayama, Japan
}

*Corresponding author

\section{Keywords \\ Biocatalyst, \\ Actinomycete, \\ Stereoselective reduction, Chiral hydroxy ester, Actinoplanes, Dactylosporangium \\ Article Info \\ Accepted: \\ 10 March 2019 \\ Available Online: \\ 10 April 2019}

\section{A B S T R A C T}

\section{Introduction}

Actinobacteria are among the most morphologically diverse prokaryotes, and are widely distributed in both terrestrial and aquatic ecosystems (Servin et al., 2007; Embley and Stackebrandt, 1994). Micromonosporaceae, a family of bacteria of the class Actinobacteria, have been isolated from diverse habitats including soil, sediments, fresh and marine water, the rhizosphere, and plant tissues. Several species belonging to Micromonosporaceae produce useful enzymes (Peczyňska-Czoch and Mordarski, 1988) and degradea variety of polysaccharides (Yeager et al., 2017) to 
produce useful secondary metabolites (AlGarni et al., 2014; Solecka et al., 2012; Bérdy, 2005; Shomura et al., 1983). These bacteria therefore have important applications in industry, biotechnology, and agriculture (de Menezes et al., 2008; Rose and Steinbüchel, 2005; Linos et al., 2000). In addition, some strains of the genera Micromonospora and Salinispora in this family are useful biocatalysts for the asymmetric reduction of various carbonyl compounds such as $\alpha$ - and $\beta$-keto esters and aromatic $\alpha$-keto amides (Ishihara et al., 2013, 2011). Thus, although these genera have thus been extensively studied for their biocatalytic activities, the potential ability of other genera in this family to serve as biocatalysts has not been investigated.

In this study, we investigated the stereoselective reduction of carbonyl compounds by members of two genera, Actinoplanes (Stackebrandt and Kroppenstedt, 1987; Couch, 1950) and Dactylosporangium (Thiemann et al., 1967), of the Micromonosporaceae family in order to identify potential novel biocatalysts (Figure $1)$.

\section{Materials and Methods}

\section{Instruments and chemicals}

Gas chromatography (GC) was performed using a GL Science GC-353 gas chromatograph (GL Science Inc., Japan) equipped with capillary columns (DB-WAX, $0.25 \mu \mathrm{m}, 0.25 \mathrm{~mm}$ x $30 \mathrm{~m}$, Agilent Technologies, USA; TC-1,0.25 $\mu \mathrm{m}, 0.25 \mathrm{~mm}$ x $30 \mathrm{~m}$, GL Science Inc.; CP-Chirasil-DEX $\mathrm{CB}, 0.25 \mu \mathrm{m}, 0.25 \mathrm{~mm} \times 25 \mathrm{~m}$, Varian Inc., USA; Gamma DEX 225, $0.25 \mu \mathrm{m}, 0.25 \mathrm{~mm} x$ $30 \mathrm{~m}$, Sigma-Aldrich Inc., USA). Ethyl pyruvate (Figure 1, 1a), diatomaceous earth (granular), and NZ amine, type A were purchased from Wako Pure Chemical
Industries Ltd. (Japan). Bacto ${ }^{\mathrm{TM}}$ malt extract, Bacto $^{\mathrm{TM}}$ yeast extract, and Difco ${ }^{\mathrm{TM}}$ soluble starch were purchased from Becton Dickinson and Co. (USA). Ethyl lactate (2a), ethyl 3methyl-2-oxobutanoate (1f), ethyl 2-oxo-4phenylbutanoate (1h), ethyl 2-hydroxy-4phenylbutanoate $(2 \mathrm{~h})$, and beef extract were purchased from Sigma-Aldrich Inc. Ethyl benzoylformate (1g), ethyl 2methylacetoacetate $(1 \mathrm{j})$, and ethyl mandelate $(2 \mathrm{~g})$ were obtained from Tokyo Chemical Industry, Co., Ltd. (Japan). Ethyl 2oxobutanoate (1b), ethyl 2-oxopentanoate (1c), ethyl 2-oxohexanoate (1d), ethyl 2oxoheptanoate (1e), 2chlorobenzoylformamide (1i), 2chloromandelamide (2i), $\beta$-hydroxy esters (2b-f), and ethyl 3-hydroxy-2methylbutanoate (2j) were prepared as described previously (Mitsuhashi and Yamamoto, 2005; Kawai et al., 1995; Nakamura et al., 1988). All the other chemicals used in this study were of analytical grade and commercially available.

\section{Microorganisms and Culture}

Actinoplanes italicus NBRC13911, Actinoplanes brasiliensis NBRC13938, Actinoplanes garbadinensis NBRC13995, Actinoplanes nipponensis NBRC14063, Actinoplanes violaceus NBRC14458, Actinoplanes ferrugineus NBRC15555, Actinoplanes capillaceus NBRC16408, Actinoplanes missouriensis NBRC102363, Actinoplanes rishiriensis NBRC108556, Actinoplanes siamensis NBRC109076,

Dactylosporangium salmoneum NBRC14103, Dactylosporangium vinaceum NBRC14181,

Dactylosporangium matsuzakiense

NBRC14259,

Dactylosporangium rosum NBRC14352, Dactylosporangium fulvum NBRC14381, Dactylosporangium sp. NBRC101297, Dactylosporangum sp. NBRC101672, 
Dactylosporangium sp. NBRC101673, Dactylosporangium sp. NBRC101730, Dactylosporangium siamense NBRC106093, Dactylosporangium maewongense NBRC106094, Dactylosporangium darangshiense NBRC109065, Dactylosporangium tropicum NBRC109071, and Dactylosporangium luridum NBRC109093

The above strains were purchased from the National Institute of Technology and Evaluation, Biological Resource Center (NBRC, Japan). These strains were maintained at $28^{\circ} \mathrm{C}$ in NBRC-recommended media $(227,228,231$, and 266) solidified with $1.5 \%(\mathrm{w} / \mathrm{v})$ agar. The 227 medium (International Streptomyces Project, ISP medium No. 2) contained $4.0 \mathrm{~g}$ of Bacto ${ }^{\mathrm{TM}}$ yeast extract, $10.0 \mathrm{~g}$ of Bacto malt extract, and $4.0 \mathrm{~g}$ of D-glucose per liter of distilled water $(\mathrm{pH} 7.3)$.

The 228 medium contained1. $0 \mathrm{~g}$ of Bacto ${ }^{\mathrm{TM}}$ yeast extract, $1.0 \mathrm{~g}$ of beef extract, $2.0 \mathrm{~g}$ of $\mathrm{NZ}$ amine, type $\mathrm{A}$, and $10.0 \mathrm{~g}$ of D-glucose per liter of distilled water $(\mathrm{pH} 7.3)$. The 231 medium contained $1.0 \mathrm{~g}$ of Bacto $^{\mathrm{TM}}$ yeast extract, $1.0 \mathrm{~g}$ of beef extract, $2.0 \mathrm{~g}$ of $\mathrm{NZ}$ amine, type $\mathrm{A}$, and $10.0 \mathrm{~g}$ of maltose per liter of distilled water ( $\mathrm{pH} 7.3)$.

The 266 medium contained $2.0 \mathrm{~g}$ of Bacto $^{\mathrm{TM}}$ yeast extract, and $10.0 \mathrm{~g}$ of Difco ${ }^{\mathrm{TM}}$ soluble starch per liter of distilled water ( $\mathrm{pH}$ 7.3). The 10 Actinoplanes strains were grown in 227 , 228,231 , and 266 media for 4 days at $25^{\circ} \mathrm{C}$ with aerobic shaking in baffled flasks in the dark, and the 14 Dactylosporangium strains were grown in 227 and 266 media for 8 days at $25^{\circ} \mathrm{C}$ with aerobic shaking in baffled flasks in the dark. The actinomycetes were harvested by filtration on filter paper (Whatman No. 4) in vacuo and washed with saline $(0.85 \% \mathrm{NaCl}$ aq.). The harvested cells were immediately used for reduction after washing with the saline.
Reduction of $\alpha$ - and $\beta$-keto esters, and an aromatic $\alpha$-keto amide using resting actinomycete cells

Saline-washed wet Actinomycete cells $(0.5 \mathrm{~g}$, dry weight approximately $0.15 \mathrm{~g}$ ) were resuspended in a arge test tube $(\phi 30 \mathrm{~mm} \mathrm{x}$ $200 \mathrm{~mm}$ ) containing $20 \mathrm{~mL}$ of saline. The substrate $(0.15 \mathrm{mmol} ; 7.5 \mathrm{mM})$ was then added, and the reaction mixture was incubated aerobically (with reciprocated shaking at 120 $\mathrm{rpm})$ at $25^{\circ} \mathrm{C}$. A portion $(0.5 \mathrm{~mL})$ of the mixture was applied to a short diatomaceous earth column ( $\phi 10 \mathrm{~mm} \times 30 \mathrm{~mm})$, extracted with diethyl ether $(5.0 \mathrm{~mL})$, and then concentrated under reduced pressure.

\section{Analysis}

The production of alcohols (Figure 1, 2aj)was measured using a GC with a DB-WAX capillary column $\left(100 \mathrm{kPa} \mathrm{He}\right.$ at $110^{\circ} \mathrm{C}: 1 \mathrm{a}$, $3.78 \mathrm{~min}$; 2a, $4.75 \mathrm{~min}$; $1 \mathrm{~b}, 4.73 \mathrm{~min}$; 2b, 5.92 $\mathrm{min}$; 1f, $4.54 \mathrm{~min} ; 2 \mathrm{f}, 6.41 \mathrm{~min} ; 120^{\circ} \mathrm{C}: 1 \mathrm{c}$, $4.84 \mathrm{~min} ; 2 \mathrm{c}, 6.45 \mathrm{~min} ; 1 \mathrm{j}, 5.54 \mathrm{~min} ; 2 \mathrm{j}$-anti, $7.62 \mathrm{~min} ; 2 \mathrm{j}-$ syn, $8.13 \mathrm{~min} ; 150^{\circ} \mathrm{C}: 1 \mathrm{~d}, 3.83$ min; 2d, $4.68 \mathrm{~min}$; 1e, $4.78 \mathrm{~min}$; 2e, $6.07 \mathrm{~min}$; $\left.180^{\circ} \mathrm{C}: 1 \mathrm{~g}, 9.01 \mathrm{~min} ; 2 \mathrm{~g}, 12.08 \mathrm{~min}\right)$ or a TC1 capillary column $\left(100 \mathrm{kPa} \mathrm{He}\right.$ at $140^{\circ} \mathrm{C}: 1 \mathrm{~h}$, $10.02 \mathrm{~min} ; 2 \mathrm{~h}, 10.96 \mathrm{~min} ; 170^{\circ} \mathrm{C}: 1 \mathrm{i}, 6.85$ min; $2 \mathrm{i}, 8.34 \mathrm{~min}$ ). The enantiomeric excess (e.e.) of the product was measured using a GC instrument equipped with an optically active CP-Chirasil-DEX CB (2a-e, 2g-h, and 2j) or a Gamma DEX 225 capillary column (2f and 2i). The e.e. was calculated using the following formula: e.e. $(\%)=\{(R-S) /(R+S)\}$ x 100 , where $R$ and $S$ are the respective peak areas of the isomer in GC analyses. The absolute configurations of the $\alpha$ - and $\beta$ hydroxy esters (2a-h and $2 \mathrm{j}$ ), and the $\alpha$ hydroxy amide (2i) were identified by comparing their retention times as determined by the GC analyses with those of authentic samples (Mitsuhashi and Yamamoto, 2005; Kawai et al., 1995; Nakamura et al., 1988). 


\section{Results and Discussion}

\section{Screening of actinomycete strains and culture media}

To determine the suitable media for liquid culture, 10 Actinoplanes and 14 Dactylosporangium strains were cultivated in several culture media, after which the wet weight of the cells was measured. All Actinoplanes strains grew poorly in the 266 medium, even after 8 days of culture, and the resulting wet cell weights were $0.1 \mathrm{~g}$ or less (see Table 1).

However, two strains, Actinoplanes ferrugineus NBRC15555 and Actinoplanes missouriensis NBRC102363, yielded more than $0.5 \mathrm{~g}$ of wet cells $/ 100 \mathrm{~mL}$ of culture in both 228 and 231 media, even though the recommended medium for NBRC15555 strain is 266 medium (Table 2).

These results suggest that the amount of available carbon is more important than the type of carbon in liquid cultures of Actinoplanes strains.

Dactylosporangium strains exhibited good growth in liquid culture. The amount of wet cells obtained when culturing in 227 medium, which contains glucose as a carbon source, was larger than when using 266 medium, which contains soluble starch. Two strains in particular, Dactylosporangium sp. NBRC101297 and NBRC101730, produced up to $1.0 \mathrm{~g}$ wet cells $/ 100 \mathrm{~mL}$ when cultured in both 227 and 266 media.

We therefore investigated the potential ability of two Actinoplanes strains (NBRC15555 and NBRC102363) and two Dactylosporangium strains (NBRC101297 and NBRC101730) to act as biocatalysts for the asymmetric reduction of carbonyl compounds.

\section{Reduction of carbonyl compounds by Actinoplanes wet cells}

Two actinomycete strains (A. ferrugineus NBRC15555 and A. missouriensis NBRC102363) that were cultivated in three media $(227,228$, and 231) were tested for their ability to reduce several carbonyl compounds (Figure 1). The results of the microbial reductions are summarized in Table 3. Both strains could reduce aliphatic and aromatic $\alpha$-keto esters (1a-h) and an aromatic $\alpha$-keto amide (1i). However, there were differences in the reduction rate and stereo selectivity of the alcohols produced that were dependent on the culture medium. The reduction rate of substrates by NBRC 15555 wet cells tended to be slightly higher when compared with NBRC1023063 wet cells. More specifically, the reduction by NBRC15555 wet cells in 227 medium produced reduction ratios of $70 \%$ or more for all nine substrates tested. We therefore tried to improve the conversion ratio and the stereo selectivity of the alcohols produced by the NBRC15555 strain by introducing additives into the reduction reaction catalyzed by NBRC15555 wet cells cultured in the 227 medium (see Table 4). Three additives were tested (glucose, sodium citrate, and soy oil), and considerable improvement was observed, especially with the addition of sodium citrate, with conversion ratios to $>99 \%$ for all substrates. Furthermore, four substrates (1a, 1c-e) were stereo specifically reduced to an e.e. $>99 \%$, and the other substrates were converted to an e.e. of nearly $90 \%$.The reduction of 2-chlorobenzoylformamide (1i), an aromatic $\alpha$-keto amide, demonstrated high stereo selectivity with all the wet cells tested (Figure 1). In particular, both the NBRC15555 and NBRC102363 wet cells cultured in 228 medium reduced $1 \mathrm{i}$ to $(R)-2 \mathrm{i}$ with a high conversion ratio and excellent stereo selectivity (>99\% e.e.). As shown in Table 5, the reduction of ethyl 2- 
methylacetoacetate $(1 \mathrm{j})$, one of the $\beta$-keto esters, by the wet cells of NBRC15555 cultured in 231 medium resulted in a conversion ratio $>99 \%$; however, the stereo selectivity (syn/antiratio and e.e.) was low and was not improved by the introduction of additives.
These results indicate that the NBRC15555 strain cultured in 227 medium is a useful biocatalyst for the asymmetric reduction of carbonyl compounds such as $\alpha$-keto esters and aromatic $\alpha$-keto amides.

Table.1 The cultivation of Actinoplanes strains in several culture medium

\begin{tabular}{|c|c|c|c|c|c|c|}
\hline \multirow[b]{2}{*}{ Scientific name } & \multirow{2}{*}{$\begin{array}{c}\text { NBRC } \\
\text { number }\end{array}$} & \multirow{2}{*}{$\begin{array}{l}\text { Recomm. } \\
\text { medium }^{1}\end{array}$} & \multicolumn{4}{|c|}{ Wet cell weight $(\mathrm{g})^{2}$} \\
\hline & & & $\begin{array}{c}227 \\
\text { medium }^{3}\end{array}$ & $\begin{array}{c}228 \\
\text { medium }^{3}\end{array}$ & $\begin{array}{c}231 \\
\text { medium }^{3}\end{array}$ & $\begin{array}{c}266 \\
\text { medium }^{3}\end{array}$ \\
\hline Actinoplanes italicus & 13991 & 227 & 0.1 & 0.3 & 0.1 & $<0.1$ \\
\hline Actinoplanes brasiliensis & 13938 & 227 & 0.2 & 0.1 & 0.4 & $<0.1$ \\
\hline Actinoplanes garbadiensis & 13995 & 227 & 0.3 & 0.1 & 0.3 & $<0.1$ \\
\hline Actinoplanes nipponensis & 14063 & 231 & 0.4 & 0.1 & 0.2 & $<0.1$ \\
\hline Actinoplanes violaceus & 14458 & 227 & 0.1 & $<0.1$ & $<0.1$ & 0.1 \\
\hline Actinoplanes ferrugineus & 15555 & 266 & 0.3 & 0.5 & 0.6 & $<0.1$ \\
\hline Actinoplanes capillaceus & 16408 & 227 & 0.3 & 0.7 & 0.1 & $<0.1$ \\
\hline Actinoplanes missouriensis & 102363 & 231 & 0.3 & 0.6 & 0.9 & $<0.1$ \\
\hline Actinoplanes rishiriensis & 108556 & 228 & 0.2 & 0.1 & 0.1 & $<0.1$ \\
\hline Actinoplanes siamensis & 109076 & 227 & 0.1 & 0.6 & 0.2 & $<0.1$ \\
\hline
\end{tabular}

${ }^{1}$ The culture medium number NBRC (NITE Biological Resource Center) culture collection recommends

${ }^{2}$ The actinomycete were grown in the liquid medium $(100 \mathrm{~mL})$ at $25^{\circ} \mathrm{C}$ for 4 days with aerobic reciprocating shaking $\left(100 \mathrm{~min}^{-1}\right)$ in baffled

500-mL flask in the dark condition

${ }^{3}$ Composition of each culture medium was described in materials and method section

Table.2 The cultivation of Dactylosporangium strains in several culture medium

\begin{tabular}{|l|c|c|c|c|}
\hline \multicolumn{1}{|c|}{ Scientific name } & NBRC & Recomm. & \multicolumn{2}{c|}{ Wet cell weight (g) $^{\mathbf{2}}$} \\
\cline { 4 - 5 } & number & medium & 227 medium $^{3}$ & 266 medium $^{3}$ \\
\hline Dactylosporangium salmoneum & 14103 & 227 & 0.7 & 0.4 \\
\hline Dactylosporangium vinaceus & 14181 & 227 & $<0.1$ & 0.2 \\
\hline Dactylosporangium matsuzakiense & 14259 & 227 & 0.2 & 0.1 \\
\hline Dactylosporangium roseum & 14352 & 227 & 1.1 & 0.4 \\
\hline Dactylosporangium fulvum & 14381 & 227 & 0.4 & $<0.1$ \\
\hline Dactylosporangium sp. & 101297 & 266 & 1.6 & 1.6 \\
\hline Dactylosporangium sp. & 101672 & 227 & 1.3 & 0.2 \\
\hline Dactylosporangium sp. & 101673 & 227 & 0.4 & $<0.1$ \\
\hline Dactylosporangium sp. & 101730 & 266 & 1.0 & 1.0 \\
\hline Dactylosporangium siamense & 106093 & 227 & 0.4 & 0.3 \\
\hline Dactylosporangium maewongense & 106094 & 227 & 1.4 & 0.3 \\
\hline Dactylosporangium darangshiense & 109065 & 227 & 0.3 & 0.1 \\
\hline Dactylosporangium tropicum & 109071 & 227 & 0.3 & 0.3 \\
\hline Dactylosporangium luridum & 109093 & 227 & 0.4 & 0.3 \\
\hline
\end{tabular}

The culture medium number NBRC (NITE Biological Resource Center) culture collection recommends

${ }^{2}$ The actinomycete were grown in the medium $(100 \mathrm{~mL})$ at $25^{\circ} \mathrm{C}$ for 8 days with aerobic reciprocating shaking $\left(100 \mathrm{~min}^{-1}\right)$ in baffled 500-mL flask in the dark condition

${ }^{3}$ Composition of each culture medium was described in materials and method section 
Table.3 The reduction of carbonyl compounds (1a-i) to the corresponding alcohols (2a-i) by two Actinoplanes strains. ${ }^{1,2,3}$

\begin{tabular}{|c|c|c|c|c|c|c|c|c|c|c|c|c|c|c|c|c|c|c|}
\hline \multirow[b]{3}{*}{ Product } & \multicolumn{9}{|c|}{ Actinoplanes ferrugineus NBRC15555 } & \multicolumn{9}{|c|}{ Actinoplanes missouriensis NBRC102363 } \\
\hline & \multicolumn{3}{|c|}{227 medium } & \multicolumn{3}{|c|}{228 medium } & \multicolumn{3}{|c|}{231 medium } & \multicolumn{3}{|c|}{227 medium } & \multicolumn{3}{|c|}{228 medium } & \multicolumn{3}{|c|}{231 medium } \\
\hline & $\begin{array}{c}\text { Conv. } \\
(\%)\end{array}$ & $\begin{array}{l}\text { e.e. } \\
(\%)\end{array}$ & $R / S$ & $\begin{array}{c}\text { Con } \\
\text { V. } \\
(\%)\end{array}$ & $\begin{array}{l}\text { e.e. } \\
(\%)\end{array}$ & $R / S$ & $\begin{array}{c}\text { Conv. } \\
(\%)\end{array}$ & $\begin{array}{l}\text { e.e. } \\
(\%)\end{array}$ & $R / S$ & $\begin{array}{c}\text { Conv. } \\
(\%)\end{array}$ & $\begin{array}{l}\text { e.e. } \\
(\%)\end{array}$ & $R / S$ & $\begin{array}{c}\text { Conv. } \\
(\%)\end{array}$ & $\begin{array}{l}\text { e.e. } \\
(\%)\end{array}$ & $R / S$ & $\begin{array}{c}\text { Conv. } \\
(\%)\end{array}$ & $\begin{array}{l}\text { e.e. } \\
(\%)\end{array}$ & $R / S$ \\
\hline $2 a$ & $>99$ & $>99$ & $S$ & 92 & 73 & $S$ & 90 & 72 & $S$ & 37 & 52 & $R$ & $>99$ & 90 & $S$ & 93 & 82 & $S$ \\
\hline $2 \mathbf{b}$ & 82 & 66 & $S$ & 80 & 46 & $S$ & 83 & 39 & $R$ & 20 & 89 & $R$ & 67 & 9 & $R$ & 32 & 63 & $R$ \\
\hline $2 c$ & $>99$ & $>99$ & $S$ & 99 & 94 & $S$ & 65 & 21 & $R$ & 27 & 70 & $R$ & 29 & 69 & $R$ & 46 & 41 & $R$ \\
\hline 2d & 89 & 54 & $S$ & 82 & 99 & $S$ & 51 & 13 & $R$ & 18 & 79 & $R$ & 70 & 11 & $R$ & 41 & 31 & $R$ \\
\hline $2 \mathrm{e}$ & 75 & 51 & $R$ & 78 & 99 & $S$ & 45 & 60 & $R$ & 11 & 95 & $R$ & 5 & 18 & $R$ & 28 & 83 & $R$ \\
\hline $2 f$ & 72 & 30 & $S$ & 49 & 19 & $S$ & 75 & 83 & $R$ & 16 & 73 & $S$ & 39 & 27 & $S$ & 74 & 33 & $R$ \\
\hline $2 \mathrm{~g}$ & 71 & 29 & $S$ & 76 & 65 & $R$ & 78 & 79 & $S$ & 9 & $>99$ & $S$ & 39 & 27 & $S$ & 14 & $>99$ & $R$ \\
\hline $2 \mathrm{~h}$ & 70 & 67 & $R$ & 28 & 65 & $S$ & 28 & 92 & $S$ & 4 & $>99$ & $S$ & 28 & 97 & $S$ & 46 & 90 & $S$ \\
\hline $2 \mathrm{i}$ & 92 & $>99$ & $R$ & $>99$ & $>99$ & $R$ & 84 & $>99$ & $R$ & 85 & $>99$ & $R$ & $>99$ & $>99$ & $R$ & 29 & $>99$ & $R$ \\
\hline
\end{tabular}

${ }^{1}$ Substrate $(0.15 \mathrm{mmol}), 0.85 \% \mathrm{NaCl}$ aq. $(20 \mathrm{~mL})$ were added to the wet cells $(0.5 \mathrm{~g})$ cultured in liquid medium, and the reaction mixture was incubated aerobically

(reciprocating shaking at $120 \mathrm{~min}^{-1}$ ) at $25^{\circ} \mathrm{C}$ for $48 \mathrm{hrs}$.

${ }^{2}$ Conversion was measured by a GLC analysis

${ }^{3}$ Enantiomeric excess (e.e.) and absolute configuration $(R / S)$ were determined by GLC analyses with optically active capillary columns 
Table.4 The reduction of 1 by A. ferrugineus NBRC15555 cultivated in 227 medium in the presence of additive. ${ }^{1,2,3}$

\begin{tabular}{|c|c|c|c|c|c|c|c|c|c|}
\hline \multirow[b]{3}{*}{ Product } & \multicolumn{9}{|c|}{ Additive } \\
\hline & \multicolumn{3}{|c|}{ D-Glucose } & \multicolumn{3}{|c|}{ Sodium citrate } & \multicolumn{3}{|c|}{ Soy oil } \\
\hline & $\begin{array}{c}\text { Con. } \\
(\%)\end{array}$ & $\begin{array}{l}\text { e.e. } \\
(\%)\end{array}$ & $R / S$ & $\begin{array}{c}\text { Conv. } \\
(\%)\end{array}$ & $\begin{array}{l}\text { e.e. } \\
(\%)\end{array}$ & $R / S$ & $\begin{array}{c}\text { Con } \\
\text { v, } \\
(\%)\end{array}$ & $\begin{array}{l}\text { e.e. } \\
(\%)\end{array}$ & $R / S$ \\
\hline $2 a$ & $>99$ & 42 & $S$ & $>99$ & $>99$ & $S$ & $>99$ & 43 & $S$ \\
\hline $2 b$ & 59 & 34 & $R$ & $>99$ & 92 & $R$ & $>99$ & 60 & $S$ \\
\hline $2 c$ & 62 & 19 & $R$ & $>99$ & $>99$ & $R$ & $>99$ & 97 & $S$ \\
\hline 2d & 74 & 13 & $R$ & $>99$ & $>99$ & $S$ & 95 & 40 & $S$ \\
\hline $2 e$ & 30 & 65 & $R$ & $>99$ & $>99$ & $S$ & 90 & 18 & $S$ \\
\hline $2 f$ & $>99$ & 19 & $S$ & $>99$ & 99 & $S$ & $>99$ & 35 & $S$ \\
\hline $2 g$ & 42 & $>99$ & $R$ & $>99$ & 90 & $S$ & 49 & 43 & $S$ \\
\hline $2 \mathrm{~h}$ & 45 & 15 & $R$ & $>99$ & 88 & $S$ & 90 & 43 & $S$ \\
\hline $2 \mathbf{i}$ & 99 & $>99$ & $R$ & $>99$ & $>99$ & $R$ & 98 & $>99$ & $R$ \\
\hline
\end{tabular}

${ }^{1}$ Substrate $(0.15 \mathrm{mmol}), 0.85 \% \mathrm{NaCl}$ aq. $(20 \mathrm{~mL})$ and additive (D-glucose and sodium citrate: $5 \mathrm{mmol}$, soy oil: $0.5 \mathrm{~mL})$ were added to the wet cells $(0.5 \mathrm{~g})$ cultured in 227 medium, and the reaction mixture was incubated aerobically (reciprocating shaking at $120 \mathrm{~min}^{-1}$ ) at $25^{\circ} \mathrm{C}$ for $48 \mathrm{hrs}$. ${ }^{2}$ Conversion was measured by a GLC analysis.

${ }^{3}$ Enantiomeric excess (e.e.) and absolute configuration $(R / S)$ were determined by GLC analyses with optically active capillary columns.

Table.5 The reduction of ethyl 2-methylacetoacetate (1j) to the corresponding alcohol (2j) by Actinoplanes strains. $1,2,3$

\begin{tabular}{|c|c|c|c|c|c|c|}
\hline \multirow[b]{2}{*}{ Strain } & \multirow[b]{2}{*}{ medium } & \multirow[b]{2}{*}{ Additive } & \multirow[b]{2}{*}{$\begin{array}{c}\text { Conv. } \\
(\%)\end{array}$} & \multirow[b]{2}{*}{ Syn / Anti } & \multicolumn{2}{|c|}{ e.e. $(\%)$} \\
\hline & & & & & $\begin{array}{c}S y n-(2 R, \\
3 S)\end{array}$ & $\begin{array}{c}\text { Anti- }(2 S, \\
3 S)\end{array}$ \\
\hline \multirow{3}{*}{ A. ferrugineus } & 227 & None & 4 & $20 / 80$ & 33 & 92 \\
\hline & 228 & None & 16 & $44 / 56$ & 38 & 45 \\
\hline & 231 & None & $>99$ & $59 / 41$ & 34 & 17 \\
\hline \multirow{3}{*}{ A. missoriensis } & 227 & None & 7 & $38 / 62$ & 30 & 78 \\
\hline & 228 & None & 95 & $41 / 59$ & 20 & 31 \\
\hline & 231 & None & 6 & $35 / 65$ & 42 & 84 \\
\hline \multirow{3}{*}{ A. ferrugineus } & 227 & D-Glucose & 10 & 33 / 67 & 40 & 80 \\
\hline & 227 & Sodium citrate & 5 & $22 / 78$ & 31 & 88 \\
\hline & 227 & Soy oil & 12 & $39 / 61$ & 44 & 71 \\
\hline
\end{tabular}

${ }^{1}$ Substrate $(0.15 \mathrm{mmol}), 0.85 \% \mathrm{NaCl}$ aq. $(20 \mathrm{~mL})$ and additive (D-glucose and sodium citrate: $5 \mathrm{mmol}$, soy oil: $0.5 \mathrm{~mL})$ were added to the wet cells $(0.5 \mathrm{~g})$ cultured in liquid medium, and the reaction mixture was incubated aerobically (reciprocating shaking at $120 \mathrm{~min}^{-1}$ ) at $25^{\circ} \mathrm{C}$ for $48 \mathrm{hrs}$.

${ }^{2}$ Conversion was measured by a GLC analysis.

${ }^{3}$ Enantiomeric excess (e.e.) and absolute configuration $(R / S)$ were determined by GLC analyses with optically active capillary columns. 
Table.6 The reduction of ethyl 2-methylacetate ( $1 \mathrm{j})$ to the corresponding alcohol $(2 \mathrm{j})$ by two Dactylosporangium strains. ${ }^{1,2,3}$

\begin{tabular}{|c|c|c|c|c|c|c|c|c|c|c|c|c|}
\hline \multirow[b]{3}{*}{ Product } & \multicolumn{6}{|c|}{ Dactylosporangium sp. NBRC101297 } & \multicolumn{6}{|c|}{ Dactylosporangium sp. NBRC101730 } \\
\hline & \multicolumn{3}{|c|}{227 medium } & \multicolumn{3}{|c|}{266 medium } & \multicolumn{3}{|c|}{227 medium } & \multicolumn{3}{|c|}{266 medium } \\
\hline & $\begin{array}{c}\text { Conv. } \\
(\%)\end{array}$ & $\begin{array}{l}\text { e.e. } \\
(\%)\end{array}$ & $R / S$ & $\begin{array}{c}\text { Conv } \\
(\dot{\%})\end{array}$ & $\begin{array}{l}\text { e.e. } \\
(\%)\end{array}$ & $R / S$ & $\begin{array}{c}\text { Conv } \\
\text { (\%) }\end{array}$ & $\begin{array}{l}\text { e.e. } \\
(\%)\end{array}$ & $R / S$ & $\begin{array}{c}\text { Conv } \\
\dot{(\%)}\end{array}$ & $\begin{array}{l}\text { e.e. } \\
(\%)\end{array}$ & $R / S$ \\
\hline $2 \mathbf{a}$ & 40 & 87 & $S$ & 51 & 79 & $S$ & 94 & 47 & $S$ & 90 & 22 & $S$ \\
\hline 2b & 29 & 53 & $S$ & 95 & 76 & $S$ & 48 & 18 & $S$ & 43 & 24 & $S$ \\
\hline $2 c$ & 23 & 42 & $S$ & 74 & 37 & $S$ & 46 & 14 & $S$ & 35 & 33 & $S$ \\
\hline 2d & 14 & 23 & $S$ & 8 & $>99$ & $S$ & 36 & 40 & $S$ & 29 & 19 & $S$ \\
\hline $2 e$ & 5 & 14 & $S$ & 3 & 1 & $S$ & 14 & 36 & $S$ & 7 & $>99$ & $S$ \\
\hline $2 f$ & 32 & 66 & $S$ & 60 & 14 & $S$ & 69 & $>99$ & $R$ & 75 & 22 & $R$ \\
\hline $2 \mathrm{~g}$ & 11 & 12 & $R$ & 10 & 25 & $R$ & 26 & 64 & $S$ & 19 & 47 & $S$ \\
\hline $2 \mathrm{~h}$ & 80 & 9 & $S$ & 4 & $>99$ & $S$ & 16 & 39 & $S$ & 7 & 28 & $S$ \\
\hline $2 \mathrm{i}$ & 25 & 25 & $R$ & 94 & 85 & $R$ & 80 & 38 & $R$ & 31 & 53 & $R$ \\
\hline
\end{tabular}

${ }^{1}$ Substrate $(0.15 \mathrm{mmol}), 0.85 \% \mathrm{NaCl}$ aq. $(20 \mathrm{~mL})$ were added to the wet cells $(0.5 \mathrm{~g})$ cultured in liquid medium, and the reaction mixture was incubated aerobically (reciprocating shaking at $120 \mathrm{~min}^{-1}$ ) at $25^{\circ} \mathrm{C}$ for $48 \mathrm{hrs}$.

${ }^{2}$ Conversion was measured by a GLC analysis.

${ }^{3}$ Enantiomeric excess (e.e.) and absolute configuration $(R / S)$ were determined by GLC analyses with optically active capillary columns.

Table.7 The reduction of carbonyl compounds (1a-i) to the corresponding alcohols (2a-i) by Dactylosporangium sp. NBRC101297 strain in the presence of additive. ${ }^{1,2,3}$

\begin{tabular}{|c|c|c|c|c|c|c|c|c|c|c|c|c|}
\hline \multirow[b]{3}{*}{ Product } & \multicolumn{6}{|c|}{227 medium } & \multicolumn{6}{|c|}{266 medium } \\
\hline & \multicolumn{3}{|c|}{ D-Glucose } & \multicolumn{3}{|c|}{ L-Glutamate } & \multicolumn{3}{|c|}{ D-Glucose } & \multicolumn{3}{|c|}{ L-Glutamate } \\
\hline & $\begin{array}{c}\text { Conv. } \\
(\%)\end{array}$ & $\begin{array}{l}\text { e.e. } \\
(\%)\end{array}$ & $R / S$ & $\begin{array}{c}\text { Conv. } \\
(\%)\end{array}$ & $\begin{array}{l}\text { e.e. } \\
(\%)\end{array}$ & $R / S$ & $\begin{array}{c}\text { Conv. } \\
(\%)\end{array}$ & $\begin{array}{l}\text { e.e. } \\
(\%)\end{array}$ & $R / S$ & $\begin{array}{c}\text { Conv. } \\
(\%)\end{array}$ & $\begin{array}{l}\text { e.e. } \\
(\%)\end{array}$ & $R / S$ \\
\hline $2 \mathbf{a}$ & 48 & 83 & $S$ & 76 & 43 & $S$ & 15 & 72 & $S$ & $>99$ & $>99$ & $S$ \\
\hline $2 b$ & 53 & 73 & $S$ & 68 & 78 & $S$ & 29 & 64 & $S$ & 82 & 69 & $S$ \\
\hline $2 c$ & 42 & 81 & $S$ & 68 & 81 & $S$ & 33 & 74 & $S$ & 92 & 68 & $S$ \\
\hline $2 d$ & 23 & $>99$ & $S$ & 32 & 72 & $S$ & 11 & 63 & $S$ & 64 & 68 & $S$ \\
\hline $2 e$ & 14 & $>99$ & $S$ & 60 & $>99$ & $S$ & 25 & $>99$ & $S$ & 5 & 54 & $S$ \\
\hline $2 f$ & 66 & 2 & $S$ & 80 & 28 & $R$ & 52 & 21 & $S$ & 90 & 30 & $R$ \\
\hline $2 \mathrm{~g}$ & 12 & 46 & $R$ & 22 & 46 & $R$ & 18 & 45 & $S$ & 34 & 54 & $R$ \\
\hline $2 \mathrm{~h}$ & 9 & 48 & $S$ & 35 & 58 & $S$ & 14 & 46 & $S$ & 34 & 45 & $S$ \\
\hline $2 \mathbf{i}$ & 48 & 83 & $R$ & 38 & 60 & $R$ & 49 & 88 & $R$ & 41 & 80 & $R$ \\
\hline
\end{tabular}

${ }^{\mathrm{I}}$ Substrate $(0.15 \mathrm{mmol}), 0.85 \% \mathrm{NaCl}$ aq. $(20 \mathrm{~mL})$ and additive (glucose and sodium hydrogen glutamate: 5 $\mathrm{mmol})$ were added to the wet cells $(0.5 \mathrm{~g})$ cultured in liquid medium, and the reaction mixture was incubated aerobically (reciprocating shaking at $120 \mathrm{~min}^{-1}$ ) at $25^{\circ} \mathrm{C}$ for $48 \mathrm{hrs}$.

${ }^{2}$ Conversion was measured by a GLC analysis.

${ }^{3}$ Enantiomeric excess (e.e.) and absolute configuration $(R / S)$ were determined by GLC analyses with optically active capillary columns. 
Table.8 The reduction of carbonyl compounds (1a-i) to the corresponding alcohols (2a-i) by Dactylosporangium sp. NBRC101730 strain in the presence of additive. ${ }^{1,2,3}$

\begin{tabular}{|c|c|c|c|c|c|c|c|c|c|c|c|c|}
\hline \multirow[b]{3}{*}{ Product } & \multicolumn{6}{|c|}{227 medium } & \multicolumn{6}{|c|}{266 medium } \\
\hline & \multicolumn{3}{|c|}{ D-Glucose } & \multicolumn{3}{|c|}{ L-Glutamate } & \multicolumn{3}{|c|}{ D-Glucose } & \multicolumn{3}{|c|}{ L-Glutamate } \\
\hline & $\begin{array}{c}\text { Conv. } \\
(\%)\end{array}$ & $\begin{array}{l}\text { e.e. } \\
(\%)\end{array}$ & $R / S$ & $\begin{array}{c}\text { Conv. } \\
(\%)\end{array}$ & $\begin{array}{l}\text { e.e. } \\
(\%)\end{array}$ & $R / S$ & $\begin{array}{c}\text { Conv. } \\
(\%)\end{array}$ & $\begin{array}{l}\text { e.e. } \\
(\%)\end{array}$ & $R / S$ & $\begin{array}{c}\text { Conv. } \\
(\%)\end{array}$ & $\begin{array}{l}\text { e.e. } \\
(\%)\end{array}$ & $R / S$ \\
\hline $2 \mathbf{a}$ & 71 & 55 & $S$ & $>99$ & 49 & $S$ & 71 & 36 & $S$ & 79 & 54 & $S$ \\
\hline $2 b$ & 25 & $>99$ & $S$ & 62 & 58 & $S$ & 59 & 45 & $S$ & 25 & 55 & $S$ \\
\hline $2 c$ & 57 & 40 & $S$ & 32 & 36 & $S$ & 64 & 27 & $S$ & 57 & 22 & $S$ \\
\hline $2 d$ & 45 & 55 & $S$ & 39 & 39 & $S$ & 58 & 22 & $S$ & 39 & 33 & $S$ \\
\hline $2 e$ & 18 & 13 & $S$ & 1 & 46 & $S$ & 25 & $>99$ & $S$ & 36 & $>99$ & $S$ \\
\hline $2 f$ & 71 & 20 & $S$ & $>99$ & 4 & $S$ & $>99$ & 18 & $R$ & 71 & 75 & $R$ \\
\hline $2 g$ & 11 & 67 & $R$ & 1 & 77 & $S$ & 28 & 67 & $R$ & 32 & 81 & $R$ \\
\hline $2 \mathrm{~h}$ & 5 & 10 & $R$ & 8 & 44 & $S$ & 31 & 42 & $S$ & 13 & 48 & $S$ \\
\hline $2 \mathbf{i}$ & $>99$ & 91 & $R$ & 95 & 27 & $R$ & 47 & 90 & $R$ & 41 & 80 & $R$ \\
\hline
\end{tabular}

${ }^{\mathrm{I}}$ Substrate $(0.15 \mathrm{mmol}), 0.85 \% \mathrm{NaCl}$ aq. $(20 \mathrm{~mL})$ and additive (glucose and sodium hydrogen glutamate: 5 $\mathrm{mmol})$ were added to the wet cells $(0.5 \mathrm{~g})$ cultured in liquid medium, and the reaction mixture was incubated aerobically (reciprocating shaking at $120 \mathrm{~min}^{-1}$ ) at $25^{\circ} \mathrm{C}$ for $48 \mathrm{hrs}$.

${ }^{2}$ Conversion was measured by a GLC analysis.

${ }^{3}$ Enantiomeric excess (e.e.) and absolute configuration $(R / S)$ were determined by GLC analyses with optically active capillary columns.

Table.9 The reduction of ethyl 2-methylacetoacetate (1j) to the corresponding alcohol ( $2 \mathrm{j})$ by two Dactylosporangium strains in the presence of additive. ${ }^{1,2,3}$

\begin{tabular}{|c|c|c|c|c|c|c|}
\hline \multirow[b]{2}{*}{ Strain } & \multirow[b]{2}{*}{ Medium } & \multirow[b]{2}{*}{ Additive } & \multirow{2}{*}{$\begin{array}{c}\text { Conv. } \\
(\%)\end{array}$} & \multirow[b]{2}{*}{ Syn / Anti } & \multicolumn{2}{|c|}{ e.e. $(\%)$} \\
\hline & & & & & $\begin{array}{c}S y n-(2 R, \\
3 S)\end{array}$ & $\begin{array}{c}\text { Anti- }(2 S, \\
3 S)\end{array}$ \\
\hline \multirow{6}{*}{$\begin{array}{l}\text { Dactylosporangium sp. } \\
\text { NBRC101297 }\end{array}$} & \multirow{3}{*}{227} & None & 18 & $10 / 90$ & $>99$ & $>99$ \\
\hline & & Glucose & 9 & $10 / 90$ & $>99$ & $>99$ \\
\hline & & Glutamate & 29 & $11 / 89$ & $>99$ & $>99$ \\
\hline & \multirow{3}{*}{266} & None & 86 & $8 / 92$ & $>99$ & $>99$ \\
\hline & & Glucose & 20 & $11 / 89$ & $>99$ & $>99$ \\
\hline & & Glutamate & 68 & $10 / 90$ & $>99$ & $>99$ \\
\hline \multirow{6}{*}{$\begin{array}{l}\text { Dactylosporangium sp. } \\
\text { NBRC101730 }\end{array}$} & \multirow{3}{*}{227} & None & 38 & $7 / 93$ & $>99$ & $>99$ \\
\hline & & Glucose & 98 & $<1 />99$ & $>99$ & -4 \\
\hline & & Glutamate & 46 & $<1 />99$ & $>99$ & -4 \\
\hline & \multirow{3}{*}{266} & None & 26 & $6 / 94$ & $>99$ & $>99$ \\
\hline & & Glucose & 63 & $<1 />99$ & $>99$ & -4 \\
\hline & & Glutamate & 50 & $<1 />99$ & $>99$ & -4 \\
\hline
\end{tabular}

${ }^{1}$ Substrate $(0.15 \mathrm{mmol}), 0.85 \% \mathrm{NaCl}$ aq. $(20 \mathrm{~mL})$ and additive (D-glucose and sodium hydrogen glutamate: $5 \mathrm{mmol}$, soy oil: $0.5 \mathrm{~mL})$ were added to the wet cells $(0.5 \mathrm{~g})$ cultured in liquid medium, and the reaction mixture was incubated aerobically (reciprocating shaking at $120 \mathrm{~min}^{-1}$ ) at $25^{\circ} \mathrm{C}$ for $48 \mathrm{hrs}$.

${ }^{2}$ Conversion was measured by a GLC analysis.

${ }^{3}$ Enantiomeric excess (e.e.) and absolute configuration $(R / S)$ were determined by GLC analyses with optically active capillary columns.

${ }^{4}$-: E.e. could not be measured because it could not detect the Anti-form 
Fig.1 The reduction of various carbonyl compounds (1a-j) to the corresponding alcohols (2a-j) by actinomycetes
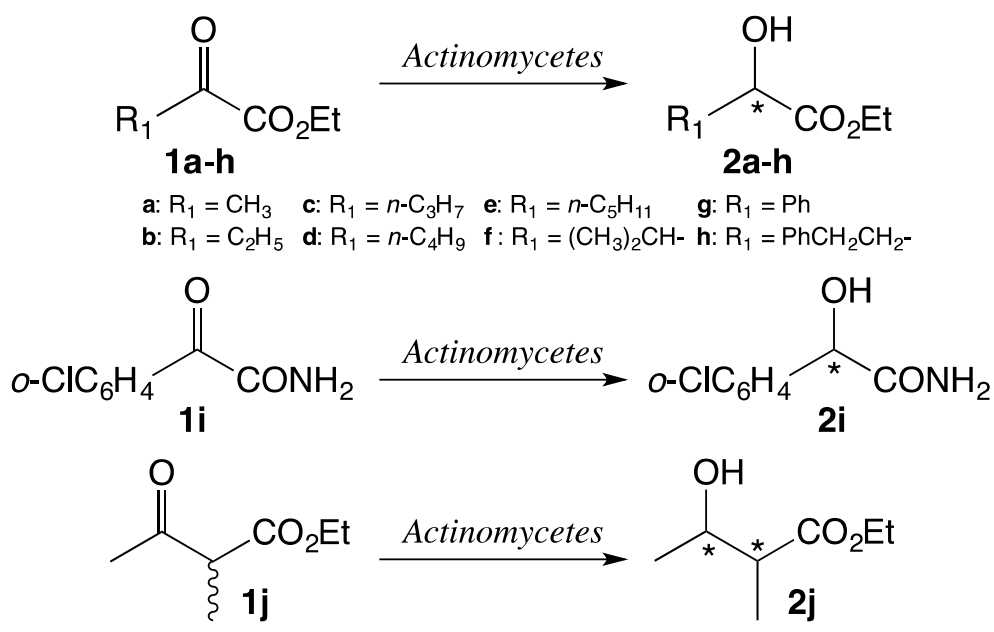

Fig.2 The reduction of $1 \mathrm{j}$ to $(2 S, 3 S)-2 \mathrm{j}$ by Dactylosporangium sp. NBRC101730 strain in the presence of glucose

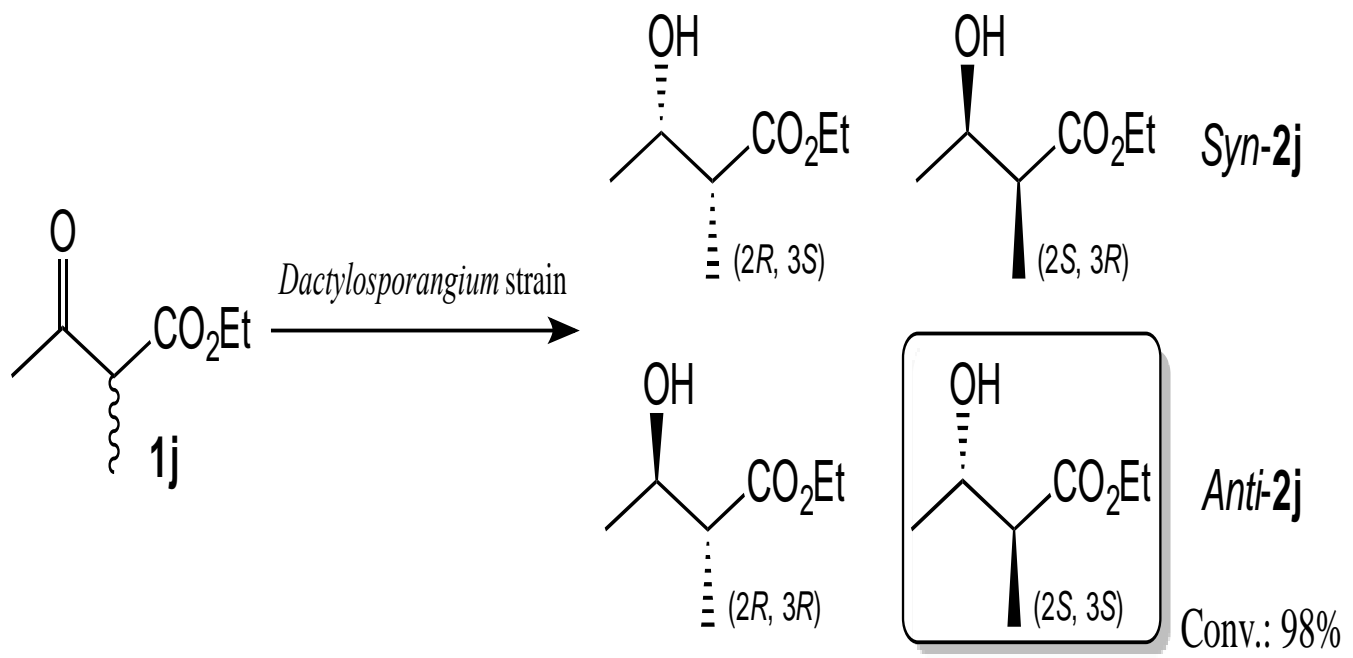

Reduction of carbonyl compounds by Dactylosporangium wet cells

Two Dactylosporangium strains cultivated in two media were tested for their ability to reduce several carbonyl compounds (Table 6). The Dactylosporangium strains could reduce aliphatic and aromatic $\alpha$-keto esters and an aromatic $\alpha$-keto amide, and no substantial difference was observed in the conversion ratio and stereoselectivity of the alcohols produced. The effects of additives on these microbial reduction reactions were also examined (Tables 7 and 8). The NBRC101297 strain cultured in 266 medium in the presence of sodium hydrogen Lglutamate reduced ethyl pyruvate (1a) to the corresponding $(S)$-alcohol (2a) with a high conversion ratio (>99\%) and excellent e.e. (> $99 \%$ ). However, the conversion rate and the 
stereoselectivity of the reduction of other substrates by same strain were not greatly improved with additive (Table 7). Similarly, the reduction efficiency and stereoselectivity by the NBRC101730 strain were not improved with additives (Table 8).

In contrast, the effect of additives on the reduction of $\beta$-keto ester was remarkable, and ethyl 2-methylacetoacetate $(1 \mathrm{j})$ reduction by NBRC101730 cultured in 227 medium in the presence of glucose stereospecifically produced the corresponding alcohol $(2 S, 3 S)$ $2 \mathrm{j}$ (Table 9). More specifically, in this reaction, the substrate was reduced to only one of the four theoretically possible isomers, in other words, this microbial reduction reaction was able to obtain a $\beta$-hydroxy ester having two chiral center carbons (Figure 2).

In conclusion, members of two genera (Actinoplanes and Dactylosporangium) from the Micromonosporaceae family were shown toconvert various $\alpha$-keto esters and an aromatic $\alpha$-keto amide to the corresponding hydroxy esters and hydroxy amide. On the basis of the conversion ratios and the stereoselectivity of the products, we suggest Actinoplanes ferrugineus NBRC15555 cultured in the 227 medium for potential use as a biocatalyst for the stereoselective reduction of $\alpha$-keto esters and the $\alpha$-keto amides to yield the corresponding chiral alcohols. Our results also suggest that the reduction of ethyl 2-methylacetoacetate by Dactylosporangium sp. NBRC101730 cultured in the 227 medium in the presence of D-glucose stereospecifically produces the corresponding chiral $\beta$-hydroxy ester.

\section{References}

Al-Garni, S.M., Sabir, J.S.M., El Hanafy, A.A.E.M., Kabli, S.A., Al-Twiley, D.A. and Ahmed, M.M. 2014. Isolation and identification of antimicrobial actinomycetes strains from Saudi environment. J. Food, Agric. Environ. 12: 1073-1079.

Bérdy, J. 2005. Bioactive microbial metabolites. J. Antibiot. 58: 1-26.

Couch, J.N. 1950 Actinoplanes, a new genus of the Actinomycetales. J. Elisha Mitchell Sci. Soc., 66: 87-92.

de Menezes, A.B., Lockhart, R.J., Cox, M.J., Allison, H.E. and McCarthy, A.J. 2008. Cellulose degradation by micromonosporas recovered from freshwater lakes and classification of these actinomycetes by DNA gryase B gene sequencing. Appl. Environ. Microbiol. 74: 7080-7084.

Embley, T.M. and Stackerbrandt, E. 1994. The molecular phylogeny and systematics of the actinomycetes. Annu. Rev. Microbiol. 48: 257-289.

Ishihara, K., Nagai, H., Takahashi, K., Nishiyama, M. and Nakajima, N. 2011. Stereoselective reduction of $\beta$ keto ester and $\beta$-keto amide with marine actinomycetes, Salinispora strains, as novel biocatalysts. Biochem. Insights 4: 29-33.

Ishihara, K., Fujita, A., Sakiyama, A., Kobayashi, U., Hori, K., Maruike, K., Masuoka, N., Nakajima, N. and Hamada, H. 2013. Preparation of chiral hydroxy esters using actinobacteria: biocatalyst activity of marine-derived Micromonospora and Streptomyces strains. Open J. Appl. Sci. 3: 116-122.

Kawai, Y., Takanobe, K. and Ohno, A. 1995. Stereochemical control in microbial reduction. XXV. Additives controlling diastereoselectivity in a microbial reduction of ethyl 2-methyl-3oxobutanoate. Bull. Chem. Soc. Jpn. 68: 285-288.

Linos, A., Berekaa, M.M., Reichelt, R., Keller, U., Scmitt, J. Flemming, H.C., Kroppenstedt, R.M., Steinbüchel, A. 
2000. Biodegradation of cis-1,4polyisoprene rubbers by distinct actinomycetes: microbial strategies and detailed surface analysis. Appl. Environ. Microbiol. 66: 1639-1645.

Mann, J. 2001. Natural products as immunosuppressive agents, Nat. Prod. Reports. 18: 417-430.

Mitsuhashi, K. and Yamamoto, H. 2005. Method for producing optically active mandelic acid derivative. Jpn. Kokai Tokkyo Koho 2005-295817 (Oct. 27).

Nakamura, K., Inoue, K., Ushio, K., Oka, S. and Ohno, A. 1988. Stereochemical control on yeast reduction of $\beta$-keto esters. Reduction by immobilized bakers' yeast in hexane. J. Org. Chem. 53: 2589-2593.

Pecznska-Czoch, W. and Mordarski, M. 1988. Actinomycete enzymes, In: Goodfellow, M., Williams, S.T. and Mordarski, M. (Ed.), Actinomycetes in biotechnology, Academic Press, London, 219-283

Rose, K. and Steinbüchel, A. 2005. Biodegradation of natural rubber and related compounds: recent insights into a hardly understood catabolic capability of microorganisms. Appl. Environ. Microbiol., 71: 2803-2812.

Servin, J.A., Herbold, C.W., Skophammer, R.G. and Lake, J.A. 2008. Evidence excluding the root of the tree of life from the actinobacteria. Mol. Biol. Evol., 25: 1-4.

Solecka, J., Zajko, J., Postek, M. and Rajnisz, A. 2012. Biologically active secondary metabolites from Actinomycetes. Central Eur. J. Biol. 7: 373-390.

Stackebandt, E. and Kroppenstedt, R.M. 1987. Union of the genera Actinoplanes couch, Ampullariella couch, and Amorphosporangium couch in a redefined genus Actinoplanes. Syst. Appl. Microbiol. 9: $110-114$

Thiemann, J.E., Pagani, H. and Beretta, G. (1967). A new genus of the Actinoplanaceae: Dactylosporangium, gen. nov. Archiv für Microbiologie. 58: $42-52$.

\section{How to cite this article:}

Ishihara, K., K. Morita, Y. Nishimori, S. Okamoto, T. Hiramatsu, A. Ohkawa, D. Uesugi, M. Yanagi, H. Hamada, N. Masuoka and Nakajima, N. 2019. Biocatalytic Reduction of Carbonyl Compounds by Actinobacteria from Two Genera of the Micromonosporaceae Family: Actinoplanes and Dactylosporangium. Int.J.Curr.Microbiol.App.Sci. 8(04): 920-931. doi: https://doi.org/10.20546/ijcmas.2019.804.106 